\title{
Urinary MMP-9/NGAL ratio as a potential marker of FSGS in nephrotic children
}

\author{
Agata Korzeniecka-Kozerska ${ }^{\mathrm{a}, *}$, Anna Wasilewska ${ }^{\mathrm{a}}$, Edyta Tenderenda ${ }^{\mathrm{a}}$, Agnieszka Sulik ${ }^{\mathrm{b}}$ and \\ Kamil Cybulski ${ }^{\mathrm{a}}$ \\ ${ }^{a}$ Department of Paediatrics and Nephrology, Medical University of Biatystok, Biatystok, Poland \\ ${ }^{\mathrm{b}}$ Department of Rheumatology and Internal Medicine, Medical University of Biatystok, Biatystok, Poland
}

\begin{abstract}
.
BACKGROUND: The study was undertaken to develop a potential new markers for distinguishing minimal change nephrotic syndrome (MCNS) and focal segmental glomerulosclerosis (FSGS) in children. We hypothesized that matrix metalloproteinase9/neutrophil gelatinase-associated lipocalin (MMP-9/NGAL) is a better marker of focal sclerosis in the glomerulus then matrix metalloproteinase-9/tissue inhibitor of metalloproteinase-1 (MMP-9/TIMP-1) and matrix metalloproteinase-2/tissue inhibitor of metalloproteinase-2 MMP2/TIMP-2.

METHODS: The present study used a sample of 36 children and adolescents subdivided into two groups: I - 20 children with MCNS, subjected to examination twice: A - in relapse of nephrotic syndrome, before treatment and B - after regression of proteinuria; II - 16 children with FSGS. MMPs and TIMPs and NGAL levels were measured in the urine using ELISA kit. MMP-9/TIMP-1, MMP-2/TIMP-2 and MMP-9/NGAL ratios were calculated.

RESULTS: Median NGAL/cr. was significantly higher in MCNS and FSGS patients when compared to healthy controls. Both, NGAL and MMP-9 urinary levels were significantly elevated in FSGS subjects, as compared with control subjects. Contrary to FSGS children, in MCNS group, before treatment only NGAL/cr., but not MMP-9/cr. was increased. Urinary concentrations of NGAL and MMP-9 were highly associated with each other (NGAL/cr. vs. MMP-9/cr., $r=0.485, p<0.01$ ). Median urine MMP-9/NGAL ratio in FSGS patients was significantly higher than in patients with MCNS. We also found that significant increase in MMP-9/NGAL was associated with FSGS [odds ratio (OR) - 9.0; confidence interval (CI) 1.97-41.07].
\end{abstract}

CONCLUSION: MMP-9/NGAL ratio may serve as differentiation marker between MCNS and FSGS in nephrotic children.

Keywords: MMP-9, NGAL, focal glomerulosclerosis, nephrotic syndrom

\section{Introduction}

Childhood nephrotic syndrome is most commonly caused by one of two idiopathic diseases: minimalchange nephrotic syndrome (MCNS) and focal segmental glomerulosclerosis (FSGS). Percutaneous renal biopsy is currently the only means of making a definite diagnosis. Biopsies are invasive, inconvenient, and sometimes can result in complications such as hemor-

${ }^{*}$ Corresponding author: Agata Korzeniecka-Kozerska, Department of Paediatrics and Nephrology, Medical University of Białystok, 15-274 Bialystok, 17 Waszyngtona Street, Poland. Tel.: +48 85 7450 663; Fax: +48 857421 838; E-mail: agatakozerska@poczta. onet.pl. rhage, infection, bleeding or gross hematuria [8]. Thus, it is important to develop a noninvasive alternative to biopsy which would decrease the likelihood of complications. We undertook this pilot study to develop potential new markers for diagnosis of FSGS in nephrotic patients.

Matrix metalloproteinases (MMPs) are a family of zinc-dependent proteinases whose activities are directed toward the degradation and turnover of extracellular matrix (ECM) proteins. Almost 30 metalloproteinases have been described to date. They are subdivided into different classes based on their structure and function [10]. Metalloproteinase-2 (MMP-2) and metalloproteinase-9 (MMP-9) degrade type IV colla- 
gen, and represent important tissue remodeling enzymes in several kidney disorders.

It has been documented that the gelatinase family of MMPs (MMP-2, MMP-9) plays the crucial role in fibrosis and chronic kidney disease progression [6,11]. MMP-2 is normally expressed in mesangial cells and podocytes during inflammation states. MMP-9 is not expressed in glomerulus in normal condition, but it's expressed in many pathological states and during development [2].

Experimental studies showed increased expression of MMP-2, but not MMP-9 in glomeruli in hypertensive glomerulosclerosis [1,7]. In humans the role of MMP-2 was confirmed in pathogenesis of tubulointerstitial fibrosis in diabetic nephropathy [11].

Expression of MMP-9 within glomeruli was found in IgA nephropathy, Schoenlein-Henoch purpura, and poststreptococcal glomerulonephritis [13].

The activity of MMP-9 is strongly regulated by a family of tissue inhibitors of metalloproteinases (TIMPs) as well as other inhibitors. One of them is neutrophil gelatinase-associated lipocalin (NGAL), a biomarker of renal injury. It was confirmed that it could bind MMP-9 and inhibit its degradation, thereby sustaining MMP-9 proteolytic activity [15]. It was found that NGAL-MMP-9 dysregulation might serve as a biomarker of diabetic nephropathy in type I DM [10].

In this pilot study, we examined urinary levels of MMP-2, MMP-9, NGAL and TIMP-1 and -2 in children with nephrotic syndrome. We hypothesized that MMP-9/NGAL is a better marker of focal sclerosis in the glomerulus then MMP-9/TIMP-1 and MMP2/TIMP-2 in children with nephrotic syndrome.

\section{Subjects and methods}

The protocol was approved by the Bioethics Committee of the Medical University of Białystok in accordance with the Declaration of Helsinki. Informed consent was obtained from parents of all participants and children older than 16 years of age.

The present study used a sample of thirty six children and adolescents ( 26 boys, 10 girls) with nephrotic syndrome hospitalized in Department of Paediatrics and Nephrology Medical University of Bialystok from January 2011 till April 2012. All nephrotic children, who fulfill the inclusion criteria were enrolled to this study. The patients were subdivided into two groups.

Group I consisted of 20 children (13 boys, 7 girls) median age: 9.0 yrs (4.3-15 yrs) with steroid-sensitive nephrotic syndrome with biopsy proven or clinically suspected MCNS subjected to examination twice: A in relapse of nephrotic syndrome, before steroid treatment and $\mathrm{B}-$ after regression of proteinuria during the prednisone therapy at a daily dose of $60 \mathrm{mg} / \mathrm{m}^{2}$ body surface area (BSA), after the mean time $8 \pm 6$ days. Diagnostic criteria for MCNS were based on the International Study of Kidney Disease in Children [10]. Inclusion criteria: children and adolescents with relapse of nephrotic syndrome with preserved renal function. After collecting the first sample, all the examined children were treated with the standard initial therapy, consisting of daily prednisone $60 \mathrm{mg} / \mathrm{m}^{2}$ BSA for 4 weeks, followed by $40 \mathrm{mg} / \mathrm{m}^{2}$ BSA given on alternate days, followed by various tapering on alternate days. Relapses were treated with daily prednisone $60 \mathrm{mg} / \mathrm{m}^{2}$ until remission was achieved, followed by $40 \mathrm{mg} / \mathrm{m}^{2}$ on alternate days.

Exclusion criteria: patients treated with other than steroids anti-inflammatory, immunomodulatory, cytostatic drugs used in the last 6 months before enrolment, patients treated with cyclosporine $\mathrm{A}$ in the past.

Group II included 16 children (13 boys, 3 girls) median age: 11 (6.5-14.5) with focal segmental glomerulosclerosis (FSGS). The frequencies of the FSGS variants in this group were: 10/16 (62.5\%) not otherwise specified (NOS), 2/16 (12.5\%) tips, 2/16 (12.5\%) collapsing, $1 / 16(6.25 \%)$ perihilar, and 1/16 (6.25\%) cellular. In group II the inclusion criteria were: patients with biopsy-proven FSGS with persistent proteinuria at least in the previous 3 months, normal renal function (estimated glomerular filtration rate $>$ $\left.90 \mathrm{ml} / \mathrm{min} / 1.73 \mathrm{~m}^{2}\right)$.

Control group (C) consisted of 32 healthy children (18 boys, 14 girls) aged median: 8.9 yrs (5.15$18.8 \mathrm{yrs})$. Younger children were diagnosed in the Department, because of isolated nocturnal enuresis. Teenagers were hospitalized, because of increased blood pressure values measured at school, which were not confirmed in ambulatory blood pressure monitoring. Health status was determined through the subjects' medical history, parental report and routine laboratory examinations to rule out the presence of acute or chronic disease. The children have never been treated with glucocorticosteroids (GCs) and protein/creatinine ratio was lower than 0.2 .

At the time of the study, the children appeared to be infection-free, with a normal body temperature $(<$ $37^{\circ} \mathrm{C}$ ) and a C-reactive protein (CRP) concentration that did not exceed $0.5 \mathrm{mg} / \mathrm{dl}$.

The clinical work-up included: the analysis of medical charts to determine the date of diagnosis, number 
Table 1

The median values and interquartile ranges of basic demographical data and examined parameters in MCNS patients, FSGS patients and healthy controls. Comparison between studied groups and controls. Significant value: ${ }^{*} p<0.05 ;{ }^{* *} p<0.01$. s.chol - serum cholesterol; s.trig. - serum triglyceride; s.crea. - serum creatinine

\begin{tabular}{lcccc}
\hline Parameters (SI) & MCNS before treatment & MCNS during treatment & FSGS \\
\cline { 2 - 5 } & \multicolumn{4}{c}{ Median (IQR) } \\
\hline Age (years) & $9.0(4.3-15)$ & $9.0(4.3-15)$ & $11^{*}(6.5-14.5)$ & $6.0(2-11)$ \\
S.chol. (mg/dl) & $212^{* *}(180-255)$ & $186^{*}(172-259)$ & $255.5^{* *}(194-394)$ & $143(134-160)$ \\
S.trig. (mg/dl) & $112(80-203)$ & $74.5(68-299)$ & $141.5(85-213)$ & $79.5(74-86)$ \\
S.crea. (mg/dl) & $0.46(0.33-0.71)$ & $0.6^{*}(0.36-0.78)$ & $0.52(0.35-0.78)$ & $0.47(0.3-0.53)$ \\
Protein/creat. ratio (mg/mg) & $12.59^{* *}(4.58-19.97)$ & $0.0(0.0-0.0)$ & $12.77^{* *}(6.28-26.37)$ & $0.0(0.0-0.0)$ \\
GFR (ml/min) & $136.98^{*}(123.4-193.2)$ & $121.84^{*}(108.2-140.1)$ & $153.59(114.2-185.6)$ & $153.78(133.1-170.71)$ \\
NGAL ng/mg cr. & $6.92^{* *}(3.94-12.74)$ & $7.21^{* *}(3.49-16.91)$ & $5.65^{*}(4.08-10.11)$ & $3.64(1.05-6.17)$ \\
MMP-2 ng/mg cr. & $0.97(0.81-2.28)$ & $1.09(0.86-1.68)$ & $1.31(0.73-2.13)$ & $7.02(5.48-11)$ \\
MMP-9 ng/mg cr. & $0.13(0.07-1.03)$ & $0.15(0.05-1.82)$ & $2.35^{* *}(0.8-7.04)$ & $0.14(0.06-0.41)$ \\
TIMP-1 ng/mg cr. & $0.65^{*}(0.15-1.25)$ & $0.14(0.0-1.38)$ & $0.86^{* *}(0.25-4.13)$ & $0.03(0.0-0.73)$ \\
TIMP-2 ng/mg cr. & $4.76^{*}(3.15-8.8)$ & $6.26(3.23-9.11)$ & $4.63(3.18-10.27)$ & $7.02(5.48-11)$ \\
MMP-9/NGAL ng/ng & $0.023(0.01-0.12)$ & $0.02(0.01-0.19)$ & $0.27^{* *}(0.12-0.88)$ & $0.13(0.02-0.18)$ \\
MMP-9/TIMP-1 ng/ng & $0.18(0.08-0.5)$ & $1.89(0.15-7.85)$ & $1.75(0.67-2.33)$ & $0.52(0.13-2.75)$ \\
MMP-2/TIMP-2 ng/ng & $0.27(0.2-0.39)$ & $0.23(0.14-0.49)$ & $0.26(0.15-0.34)$ & $0.19(0.14-0.31)$ \\
\hline
\end{tabular}

of relapses, pathological findings, previous treatment, doses of prednisone, the measurement of blood pressure and physical examination. The diagnostic studies that were reviewed included: serum and urine creatinine concentrations measured by Jaffe reaction, levels of triglycerides (TG) and total cholesterol (TC) were measured by means of colorimetric method on the Hitachi apparatus. Albumin level was determined by colorimetric method with bromcresol green, while urinalysis, urinary protein was measured by the biuret method and protein to creatinine ratio in first morning sample was calculated. Glomerular filtration rate $\left(\mathrm{ml} / \mathrm{min} / 1.73 \mathrm{~m}^{2}\right)$ was estimated by the Schwartz formula $(\mathrm{eGFR})=k x G(\mathrm{~cm}) / \mathrm{Lcr} .(\mathrm{mg} / \mathrm{dl})$, where $k$ - age - dependent coefficient $(0.55$ in boys under 12 years and girls at any age, 0.7 in boys over $12 \mathrm{yrs}$ ), $G$ - growth, Lcr. - level of creatinine in serum.

Urine measurements of the investigated markers were performed in frozen samples. Urine samples were centrifuged at $2000 \times g$ for $5 \mathrm{~min}$, and the supernatants stored in aliquots at $-80^{\circ} \mathrm{C}$. Patients with pathological leukocyturia were excluded from the examination. Control urinary samples were collected from healthy children in the same way.

\subsection{MMPs and TIMPs ELISA assay}

MMPs and TIMPs levels were measured in the urine using ELISA commercial available kit (R\&D Systems) according to manufacturer instructions. All specimens were diluted to obtained concentration for the optimal density according to ELISA kit instructions. The enzymatic reactions were quantified in an automatic microplate photometer. The detection limits were $0.16 \mathrm{ng}$ for MMP-2, $0.156 \mathrm{ng} / \mathrm{ml}$ for MMP-9, $0.08 \mathrm{ng} / \mathrm{ml}$ for TIMP-1, and $0.011 \mathrm{ng} / \mathrm{ml}$ for TIMP-2. In each sample, creatinine concentration was determined simultaneously, and the urinary excretion of MMP and TIMP was expressed as a ratio with creatinine (ng/mg cr.). MMP-9/TIMP-1 and MMP-2/TIMP-2 ratios were calculated. The mean intra- and inter- assay coefficients of variation were $5.7 \%$ and $8.2 \%$ for MMP-2, $2.0 \%$ and $7.9 \%$ for MMP-9, $4.2 \%$ and $3.9 \%$ for TIMP-1, and $4.2 \%$ and $7.8 \%$ for TIMP-2, respectively.

\subsection{NGAL ELISA assay}

NGAL was measured in the urine using ELISA commercial available kit (BioPorto Diagnostics), according to manufacturer instructions. All specimens were diluted to obtained concentration for the optimal density according to ELISA kit instructions. The enzymatic reaction was quantified in an automatic microplate photometer. Urinary NGAL level were expressed in $\mathrm{ng} / \mathrm{ml}$. The detection limit was $<0.1 \mathrm{ng} / \mathrm{ml}$.

Urinary creatinine concentration was used to normalize NGAL measurements to account for the influence of urinary dilution on its concentration. The urinary levels of creatinine were analyzed by Jaffé's method. Urinary levels of NGAL (uNGAL) were expressed as urinary NGAL/creatinine ratio in $\mathrm{ng} / \mathrm{mg}$ cr.

Data analysis was performed using computer program Statistica 10.0. Data are reported as medians and inter-quartile ranges. The nonparametric statistics were chosen as the patient population of this study was relatively small. Statistical analysis was performed using the Mann-Whitney U test, Wilcoxon test or Friedman's ANOVA when appropriate. Spearman correlation coef- 


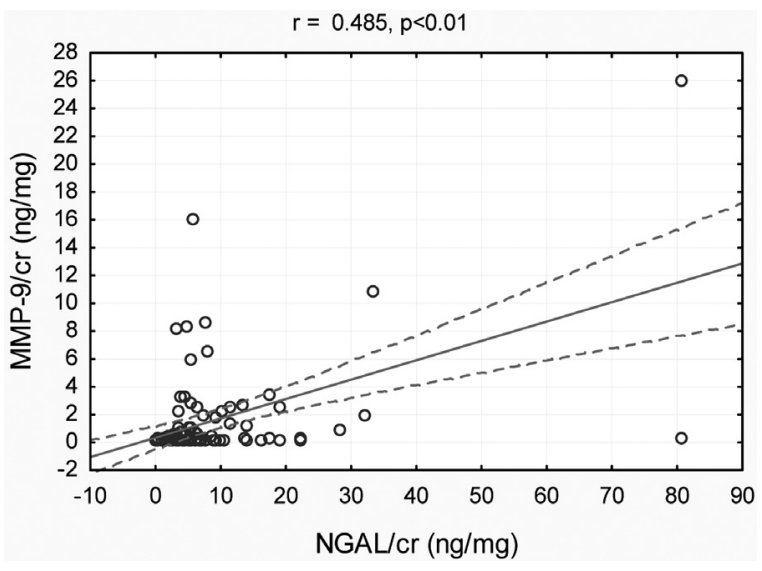

Fig. 1. Correlation between MMP-9/cr. and NGAL/cr. in patients with FSGS. Significant value $p<0.05$.

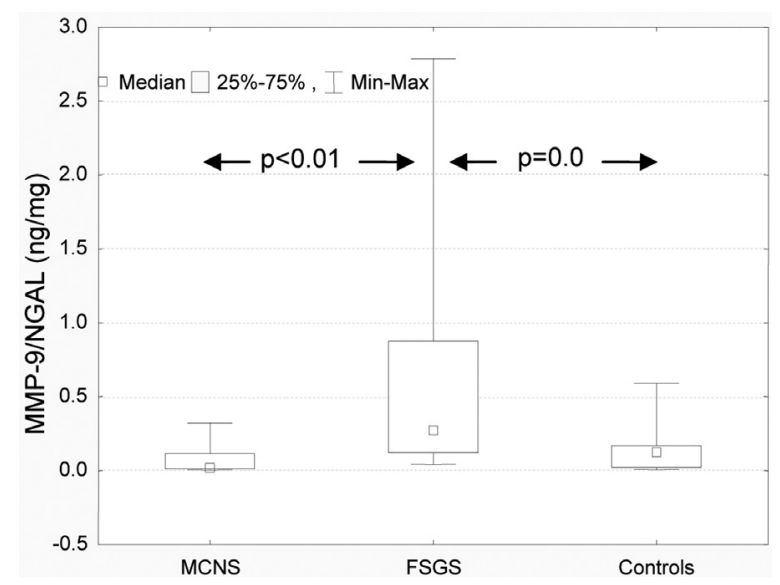

Fig. 2. Urinary MMP-9/NGAL median levels in patients with MCNS in relapse, before treatment, FSGS and comparison between both patient group and health controls. Significant value $p<0.05$.

ficients were calculated for statistically significant correlation. A $p$ value less than 0.05 was considered statistically significant.

\section{Results}

The basic characteristics of the study subjects are presented in Table 1. Children with FSGS were older then children with MCNS ( $p=0.024)$ and healthy controls. Serum triglyceride and creatinine concentration values did not differ significantly among the MCNS patients and the controls $(p>0.05)$ and among both study groups $(p>0.05)$. Both FSGS and MCNS groups differed from controls with respect to serum cholesterol $(p<0.01)$ and protein/creatinine ratio $(p<$
0.01). No statistically significant differences in these parameters were found between MCNS and FSGS patients before treatment $(p>0.05)$.

We found statistically significant increased MMP9/cr. and TIMP-1/cr. in FSGS children and TIMP-1/cr. in MCNS when compared to the controls $(p<0.01)$. Median NGAL/cr. was significantly higher in MCNS and FSGS patients when compared to healthy controls. Median NGAL/cr. did not differ significantly between MCNS and FSGS patients.

In further analysis, we found that urinary concentrations of NGAL and MMP-9 were highly associated with each other (NGAL/cr. vs. MMP-9/cr., $r=0.485$, $p<0.01$ ) (Fig. 1).

In order to evaluate the role of urine concentration of metalloproteinases and their inhibitors in distinguishing glomerular sclerosis, we compared the MMP-9/TIMP-1, MMP-9/NGAL and MMP2/TIMP2 in urine of nephrotic children in dependence of histopathological diagnosis and healthy controls. The differences observed in the median urine values of MMP-9/NGAL ratio among the MCNS and FSGS patient and healthy controls were significant $(p<0.01$, ANOVA test) (Fig. 2). Further statistical analysis using multiple comparisons between groups revealed that the median urine MMP-9/NGAL (0.27 IQR (0.12-0.88) in FSGS patients, were significantly higher than in patients with MCNS, before the treatment (0.023 IQR $(0.01-0.12)(p<0.01)$ and healthy controls $(0.13$ IQR $(0.02-0.18)(p=0.0)$. No statistically significant differences were found in MMP-9/NGAL ratio between MCNS patients and controls. The median MMP9/NGAL ratio was higher in MCNS patients in relapse then in remission, but failed to reach statistical significance (Wilcoxon test). No significant relationship between MMP-9/NGAL and histological variants of FSGS were found, however it might be influenced by the sample size.

The MMP-9/NGAL ratio demonstrated a significant $(p<0.05)$ positive correlation with creatinine level $(r=0.45)$. No correlation between MMP-9/NGAL ratio and proteinuria (protein/cr. ratio) was found.

We found that significant increase in MMP-9/NGAL (above median value in controls) was associated with focal segmental glomerulosclerosis [odds ratio (OR) 9.0; confidence interval (CI) 1.97-41.07].

\section{Discussion}

Our study describes for the first time the urinary MMP-9/NGAL ratio, as a marker of FSGS in chil- 
dren with nephrotic syndrome. The role of urinary levels of MMP-2, MMP-9 and their tissue inhibitors TIMP-1 and TIMP-2 in nephrotic children were analysed in our previous study [14] in children with MCNS treated with cyclosporine A. We did not find any significant differences in MMP-9/TIMP-1 ratio and MMP-2/TIMP-2 ratio in MCNS children, before cyclosporine treatment. Czech et al. [3] found that urinary concentrations of MMP-2, MMP-9, TIMP-1, and TIMP-2 were significantly elevated in focal segmental glomerulosclerosis as compared to steroid sensitive nephrotic syndrome in both relapse and remission.

Most studies in the literature have focused on NGAL and MMP-9/NGAL tissue expression and only a few have reported the urinary measurements. It was found that NGAL and MMP-9 are stored in specific granules in neutrophils, while MMP-9 is also found in gelatinase granules. They both mainly exist in forms not associated with each other [15]. MMP-9/NGAL complex is large, so it seems unlikely that it can be directly filtered from serum to urine. That is why it has been suggested that MMP-9 and NGAL are secreted in blood by neutrophils and are separately excreted in urine where they subsequently form complexes [16].

The important finding of this study is the fact that urine MMP-9/cr. level was significantly higher in FSGS children when compared with MCNS; however we have not confirmed this relation as regards to MMP2, TIMP-1 and TIMP-2. Zakiyanov et al. [17] reported unchanged levels of plasma MMP-2 and MMP-9 in MCNS/FSGS patients.

Similarly, the urinary concentration of NGAL was measured in nephrotic children. In our previous study in nephrotic children we found increased NGAL/cr. ratio in relapse of minimal change nephrotic syndrome, before cyclosporine treatment. In present study urine NGAL/cr. ratio was increased in both patients groups when compared to healthy controls.

Even in MCNS patients in remission of proteinuria, NGAL/cr. ratio was significantly higher. However we have not found statistically significant difference between FSGS and MCNS group of patients. However to our best knowledge it is the first report on MMP9/NGAL ratio in relation to histological changes.

In MCNS children the urine MMP-9/NGAL ratio both in relapse of nephrotic syndrome, before steroid therapy and in remission was similar when compared to healthy controls. However, it was more than 10 times higher in FSGS patients than in MCNS before treatment and in remission. We also demonstrated strong positive correlation between urine MMP-9/cr. and NGAL/cr. MMP-9/NGAL complexes have been identified in normal urine [16]. The strong association between NGAL and MMP-9 in urine suggests that these ligands may be co-excreted in nephrotic syndrome.

The urine MMP-9/NGAL complex system was studied in children with acute cystitis [5]. Thraillkill et al. [12] measured both urine MMP-9 and NGAL and found positive correlation with glycemic control and albuminuria. In our study we have not found any correlation between the MMP-9/NGAL ratio and serum albumin or urine protein/creatinine ratio.

We were also concerned whether the relapse in MCNS may influence urine MMP9/NGAL ratio, but we have not found any significant difference. It is also noteworthy that all the patients included in the study had not been treated with cytostatic drugs at least 6 months before the study. Children treated with cyclosporine were excluded from the study, because of its possible influence on NGAL and MMP-9 excretion documented in previous studies [14].

In the present study we demonstrated that MMP9/NGAL ratio was significantly increased in FSGS children and we therefore conclude that it may serve as differentiation marker between MCNS and FSGS in nephrotic children.

However we are aware of the limitation of our study. First, this was a single-center, cross-sectional pilot study with a small group of patients who had already begun treatment at enrolment. Another important point is the fact that MMP-9 activity diminishes with age [9], however in our study the age of patients with FSGS was higher, so it was not a confounder. We have not biopsied all patients with steroid-sensitive nephrotic syndrome, so we classified them as "MCNS", but we cannot say this with scientific certainty.

Secondly, since the data are not normally distributed, we used non-parametric statistics to analyze our data. Additionally, the functions of NGAL are still not fully understood. It appears to be up-regulated in cells under "stress" from different factors, e.g. infection, inflammation, and ischemia. So it is apparent that a variety of independent pathologies are associated with raised levels of urinary NGAL. Therefore the finding of a raised level cannot be independently diagnostic. Its complex formation with MMP-9 appears to protect MMP-9 enzymatic activity from degradation [16]. In our study MMP-9/NGAL seems to be of greater value than NGAL/cr. alone. Similarly the direct source of urinary MMP-9 excreted in urine cannot be determined. 
In conclusion the results of this pilot study should be further confirmed by the observation of a larger number of patients in multicenter study.

\section{References}

[1] J. Bolbrinker, S. Markovic, M. Wehland, W.B. Malenhorst, H. van Goor and R. Kreutz, Expression and response to angiotensin-converting enzyme inhibition of matrix metalloproteinases 2 and 9 in renal glomerular damage in young transgenic rats with rennin-dependent hypertension, J Pharmacol Exp Ther 316 (2006), 8-16.

[2] H.R. Chang, S.F. Yang, M.L. Li, C.C. Lin, Y.S. Hsieh and J.D. Lian, Relationships between circulating matrix metalloproteinase- 2 and -9 and renal function in patients with chronic kidney disease, Clin Chim Acta 366 (2006), 243-248.

[3] K.A. Czech, M. Bennett and P. Devarajan, Distinct metalloproteinase excretion patterns in focal segmental glomerulosclerosis, Pediatr Nephrol 26 (2011), 2179-2184.

[4] A.A. Eddy and J.M. Symons, Nephrotic syndrome in childhood, Lancet 362 (2003), 629-639.

[5] S. Hatipoglu, E. Sevketoglu, A. Gedikbasi, A. Yilmaz, A. Kayak, M. Mulazimgoglu, G. Aydogan and T. Ozpacaci, Urinary MMP-9/NGAL complex in children with acute cystitis, Pediatr Nephrol 26 (2011), 1263-1268.

[6] J. Keeling and G.A. Herrera, Human matrix metalloproteinases: Characteristics and pathologic role in altering mesangial homeostasis, Microsc Res Tech 71 (2008), 371379.

[7] S. Liu, Y. Li, H. Zhao, D. Chen, Q. Huang, S. Wang, W. Zou, Y. Zhang, X. Li and H. Huang, Increase in extracellular crosslinking by tissue transglutaminase and reduction in expression of MMP-9 contribute differentially to focal segmental glomerulosclerosis in rats, Mol Cell Biochem 284 (2006), 917.

[8] C. Manno, G.F. Strippoli, L. Arnesano, C. Bonifati, N. Campobasso, L. Gesualdo and F.P. Schena, Predictors of bleeding complications in percutaneous ultrasound-guided renal bio- psy, Kidney Int 66 (2004), 1570-1577.

[9] J.J. Marler, S.J. Fishman, S.M. Kilroy, J. Fang, J. Upton, J.B. Mulliken, P.E. Burrows, D. Zurakowski, J. Folkman and M.A. Moses, Increased expression of urinary matrix metalloproteinases parallels the extend and activity of vascular anomalies, Pediatrics 116 (2005), 38-45.

[10] C. Tallant, A. Marrero and F.X. Gomis Ruth, Matrix metalloproteinases: Fold and function of their catalytic domains, Biochim Biophys Acta 1803 (2010), 20-28.

[11] K.M. Thrailkill, R. Clay Bunn and J.L. Fowlkes, Matrix metalloproteinases: Their potential role in pathogenesis of diabetic nephropathy, Endocrine 35 (2009), 1-10.

[12] K.M. Thrailkill, C.S. Moreau, G.E. Cockrell, C. H. Jo, R.C. Bunn, A.E. Morales-Pozzo, C.K. Lumpkin and J.L. Fowlkes, Disease and gender-specific dysregulation of NGAL and MMP-9 in type 1 diabetes mellitus, Endocrine 37 (2010), 336343.

[13] M. Urushihara, S. Kagami, T. Kuhara, T. Tamaki and Y. Kuroda, Glomerular distribution and gelatinolytic activity of matrix metalloproteinases in human glomerulonephritis, Nephrol Dial Transplant 17 (2002), 1189-1196.

[14] A. Wasilewska and W. M. Zoch Zwierz, Urinary levels of matrix metalloproteinases and their tissue inhibitors in nephritic children, Pediatr Nephrol 23 (2008), 1795-1802.

[15] X. Provatopoulou, A. Gounaris, E. Kalogera, F. Zagouri, I. Papassotiriou and G. Zografos, Circulating levels of matrix metalloproteinase-9 (MMP-9), neutrophil gelatinaseassociated lipocalin (NGAL) and their complex MMP9/NGAL in breast cancer disease, BMC Cancer 9 (2009), 390.

[16] L. Yan, N. Borregaard, L. Kjeldsen and M.A. Moses, The high molecular weight urinary matrix metalloproteinase (MMP) activity is a complex of gelatinase B/MMP-9 and neutrophil gelatinase-associated lipocalin (NGAL). Modulation of MMP-9 activity by NGAL, J Biol Chem 276 (2001), 37258-37265.

[17] O. Zakiyanov, M. Kalousova, M. Kratochwilova, V. Kriha, T. Zima and V. Tesar, Changes in levels of matrix metalloproteinase-2 and -9 , pregnancy-associated plasma protein-A in patients with various nephropathies, J Nephrol (2012), DOI: 10.5301/jn.5000136. 


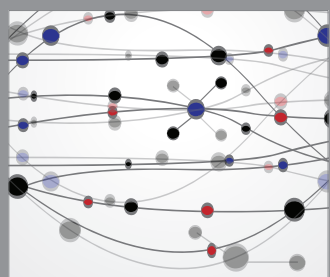

The Scientific World Journal
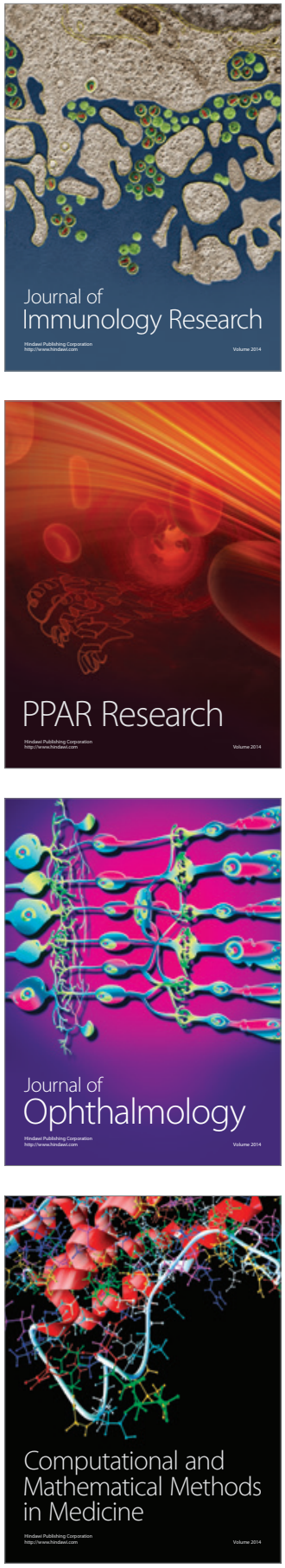

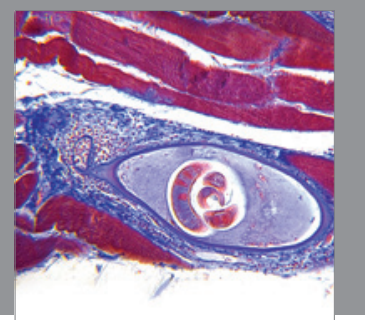

Gastroenterology

Research and Practice
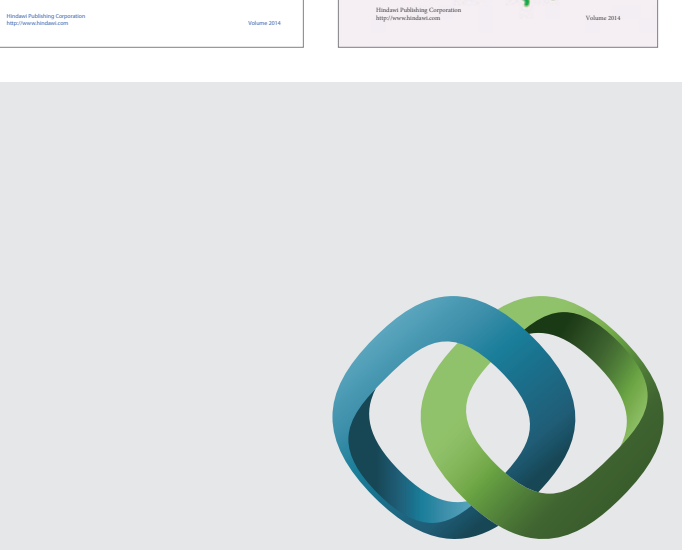

\section{Hindawi}

Submit your manuscripts at

http://www.hindawi.com
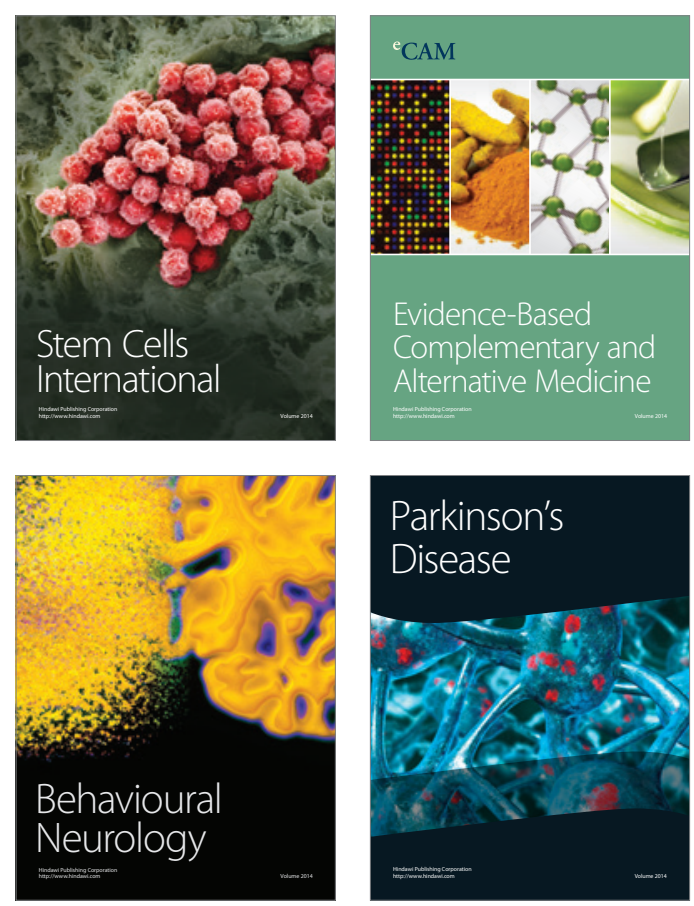

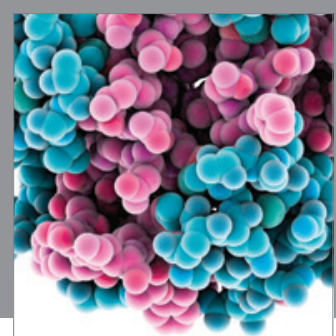

Journal of
Diabetes Research

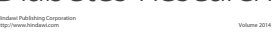

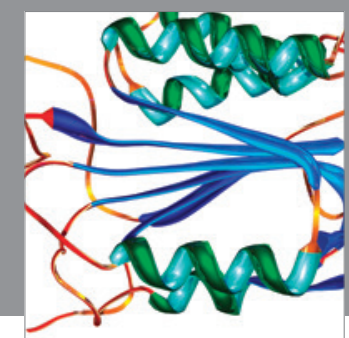

Disease Markers
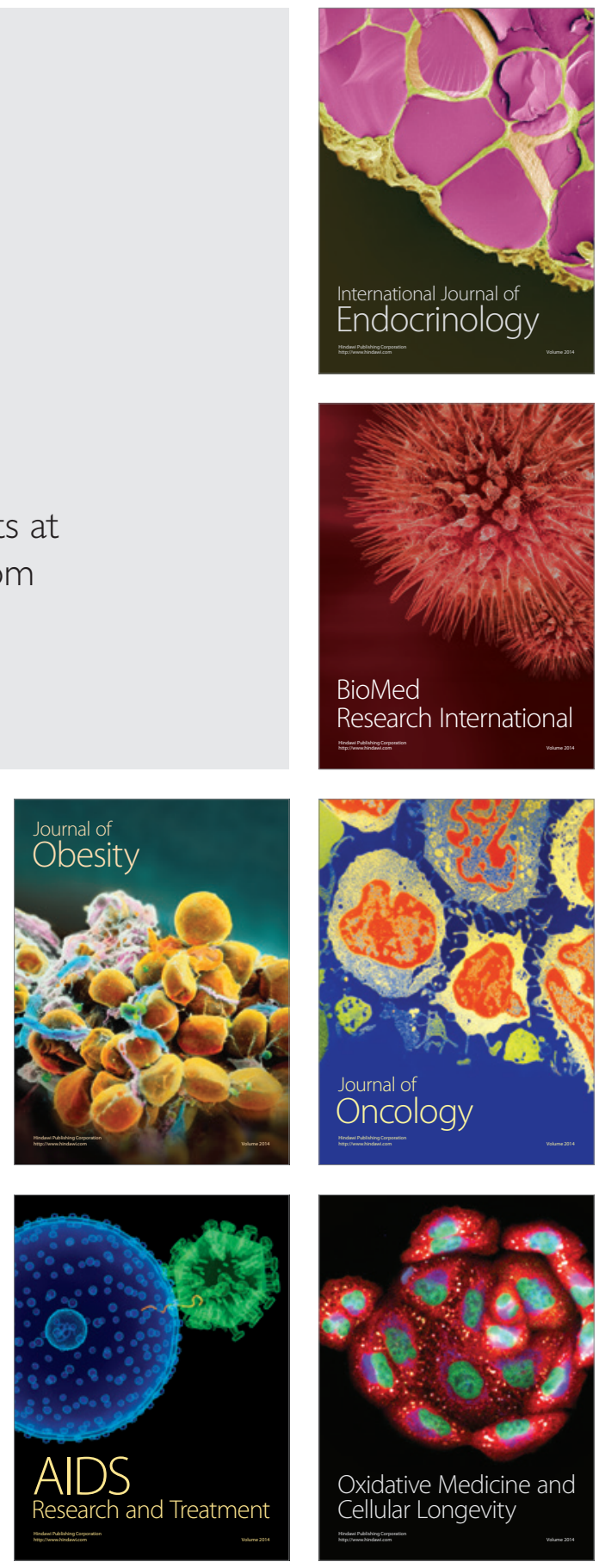